\title{
Adaptive Code Allocation for Interference Management on the Downlink of DS-CDMA Systems
}

\author{
Emad Alsusa, Senior Member, IEEE, and Christos Masouros, Student Member, IEEE
}

\begin{abstract}
A new technique based on adaptive code-to-user allocation for interference management on the downlink of BPSK based TDD DS-CDMA systems is presented. The principle of the proposed technique is to exploit the dependency of multiple access interference on the instantaneous symbol values of the active users. The objective is to adaptively allocate the available spreading sequences to users on a symbol-by-symbol basis to optimize the decision variables at the downlink receivers. The presented simulations show an overall system BER performance improvement of more than an order of a magnitude with the proposed technique while the adaptation overhead is kept less than $10 \%$ of the available bandwidth.
\end{abstract}

Index Terms-Adaptive signal processing, DS-CDMA, code allocation.

\section{INTRODUCTION}

$\mathbf{T}$ HE use of orthogonal Walsh-Hadamard spreading sequences provides excellent performance for the downlink of direct sequence code division multiple access (DSCDMA) systems in additive white Gaussian noise (AWGN) channels. However, the hostile nature of the wireless channel can severely degrade the orthogonality of such sequences and unless compensated for at the receiver it will result in significant multiple access interference (MAI). Optimizing the signature waveforms for transmission in DS-CDMA can greatly benefit a wireless communication system. Many researchers have proposed optimization of the spreading codes towards orthogonalizing the users in multipath scenarios which involved waveform design of the codes used taking into account the characteristics of the channel encountered (see e.g. [1], [2] \& [3]). This letter proposes a different approach to code optimization in which, instead of performing code waveform design, the spreading sequences available in the system are used unmodified but are adoptively allocated to the users on a symbol-by-symbol basis. In addition, instead of total interference rejection, as adopted in conventional techniques, a secondary objective of the adaptive code-touser allocation is to influence and exploit the constructive interference inherent in the system to deliver an enhanced signal to interference-plus-noise ratio (SINR) at the receiver. Constructive is the component of MAI that increases the desired symbol's energy, while destructive is the MAI that decreases the symbol's energy. More specifically, the MAI experienced by the different users depends on the crosscorrelations of the users' codes as well as the instantaneous

Manuscript received December 8, 2006; revised August 3, 2007; accepted May 8, 2008. The associate editor coordinating the review of this paper and approving it for publication was M. Chiang.

The authors are with the School of Electrical \& Electronic Engineering, The University of Manchester, PO Box 88, Manchester M16 1QD UK (e-mail: e.alsusa@manchester.ac.uk, chris.masouros@postgrad.manchester.ac.uk).

Digital Object Identifier 10.1109/TWC.2008.061043. values of the users' data symbols to be transmitted. Hence, by appropriately redistributing the codes and consequently the cross-correlation values amongst the users taking into account the values of the data symbols to be transmitted at each symbol period, MAI can be manipulated. The reallocation is done in such way that the destructive component of MAI is minimized while constructive component is enhanced to provide optimized decision variables at the mobile unites' (MUs') receivers towards making detection more reliable. This is the objective here and constitutes the adaptation criteria of the proposed method. It should be noted that with this method the improvement in the received SINR is attained without the need for additional per-user-power investment at the transmitter, as energy inherent in the system is exploited.

It may be clear by now that the proposed technique entails some overhead in the form of transmitting side information (SI) in order to inform the MUs' receivers of their code allocation at each symbol period to achieve correct despreading. This paper presents an efficient way of encoding such overhead and it will be demonstrated in the results section that the bandwidth efficiency reduction due to the SI transmission can be maintained at less than $10 \%$ of the total bandwidth. This is worthwhile as the achieved overall system bit error rate (BER) improvements are more than an order of a magnitude compared to the non-adaptive case.

\section{SYSTEM DESCRIPTION}

Consider the downlink transmission of a synchronous CDMA system of $K$ users under frequency selective fading, where the channels' path delays are assumed to be an integer number of the chip period. All codes and channels are assumed to have normalized energy of one and length of $L$ and $P$ chips, respectively. The data frame is $N$ symbols long. $T_{b}$ and $T_{c}$ are the symbol and chip periods, respectively. The received signal at the $u-t h \mathrm{MU}$ can be expressed as

$r_{u}(t)=\sum_{i=1}^{N} \sum_{k=1}^{K} \sum_{p=1}^{P} a_{k} x_{k}(i) c_{k}\left(t-i T_{b}-p T_{c}\right) h_{p u}(i)+n_{u}(t)$

where $x_{k}(i), a_{k}, c_{k}$ are the $k-t h$ user's PSK modulated data symbol for the $i-t h$ symbol period, amplitude and code, $h_{p u}(i)$ and $n_{u}(t)$ are the $u-t h$ MU's channel $p-t h$ tap coefficient and AWGN noise corrupting the signal of interest. The output of the Rake receiver of the $u-t h$ user can be expressed as

$$
\begin{aligned}
d_{i u} & =\sum_{p=1}^{P} \int_{i T_{b}+p T_{c}}^{(i+1) T_{b}+p T_{c}} r_{u}(t) h_{p u}^{*}(i) c_{u}^{*}\left(t-i T_{b}-p T_{c}\right) d t \\
& =a_{u} x_{i u}+I C I_{i u}+I S I_{i u}+M A I_{i u}+\eta_{i u}
\end{aligned}
$$


Here $x_{i u}$ is a compact representation of the desired $(u-t h)$ user's signal for the $i-t h$ period of interest, $I C I_{i u}$ is the InterChip Interference between adjacent chips, $I S I_{i u}$ is the InterSymbol Interference caused by adjacent symbols, $M A I_{i u}$ is the cumulative MAI caused by the interfering $K-1$ users and $\eta_{i u}$ is the noise component at the Rake output. If a discrete time representation is adopted by sampling the signals at the chip rate with rectangular pulses then $T_{b}, T_{c}$ can be omitted and the above terms can be defined as

$$
\begin{aligned}
I C I_{i u} & =a_{u} x_{i u}+\sum_{l=1}^{L+P-1} s_{u}[l] s_{u}^{*}[l]-a_{u} x_{i u} \\
I S I_{i u} & =a_{u} \sum_{\substack{n=-i \\
n \neq i}}^{N-i} x_{i u} \cdot \sum_{l=1}^{L+P-1} s_{u u}[l] s_{u u}^{*}[l+n L] \\
M A I_{i u} & =\sum_{n=-i}^{N-i} \sum_{\substack{k=1 \\
k \neq u}}^{K} a_{k} x_{i k} \cdot \sum_{l=1}^{L+P-1} s_{k u}[l] s_{u u}^{*}[l+n L] \\
& =\sum_{n=1}^{N} \sum_{\substack{k=1 \\
k \neq u}}^{K} a_{k} x_{i k} \rho_{k u n} \\
& s_{k u}[l]=\sum_{p=1}^{P} c_{k}[l-p+1] \cdot h_{u}[p]
\end{aligned}
$$

where $h_{u}[p]$ is the discrete time representation of $h_{p u}(i)$ and

$$
\rho_{k u n}=\sum_{l=1}^{L+P-1} s_{k u}[l] s_{u u}^{*}[l+n L]
$$

is the cross-correlation of the users' multipath-corrupted signature waveforms $\left(s_{k u}\right)$ in (6). Evidently, even if orthogonal codes are used the resulting crosscorreltation of the codes viewed at the receiver is non-zero due to the channel distortion.

For reasons of simplicity, in the following we adopt a bitwise approach but expansion to blockwise processing as in [4] is straightforward. The index $n$ in of (7) can consequently be dropped. Furthermore, it should be clarified that, as in the majority of conventional precoding schemes (e.g. in $[1,4])$, the knowledge of the channel response for all receivers is required at the base station (BS) for the proposed precoding. This can be made available by channel estimation at the transmitter in the time division duplex (TDD) transmission mode [5], which is assumed in this paper. It can be seen in (2) that given the channel state information (CSI) and data knowledge readily available at the BS the decision variables at the receiver can be pre-estimated. By selecting the appropriate code allocation for transmission at each symbol period the factors can be influenced and hence the distribution of the $d_{i u}$ values in (2) for all users can be improved to offer enhanced reliability in the detection. To clarify this we present a simple example of $K=5$ users as shown in Table 1 . Here the distributions of the decision variables for eight different code allocation patterns are depicted, for two different transmitted symbols combinations. The available spreading sequences and the allocation patterns are also shown for reasons of completeness. It can be seen that by changing the code-to-user allocation
TABLE I

NOISELESS DECISION VARIABLES' DISTRIBUTIONS FOR DIFFERENT ALLOCATION PATTERNS OF A SYSTEM OF $K=5$ USERS WITH RANDOM CODES OF $L=16$ FOR $p_{c}=8$

\begin{tabular}{|l|c|c|c|c|c|}
\cline { 2 - 6 } \multicolumn{1}{c|}{} & $x_{1}$ & $x_{2}$ & $x_{3}$ & $x_{4}$ & $x_{5}$ \\
\hline allocation pattern & -1 & -1 & 1 & -1 & 1 \\
\cline { 2 - 6 }$\left[c_{1}, c_{2}, c_{3}, c_{4}, c_{5}\right]$ & $d_{1}$ & $d_{2}$ & $d_{3}$ & $d_{4}$ & $d_{5}$ \\
\hline$s=1,\left[c_{E}, c_{A}, c_{D}, c_{C}, c_{B}\right]$ & -0.75 & -1.5 & 1.25 & -1 & 1 \\
\hline$s=2,\left[c_{A}, c_{D}, c_{C}, c_{E}, c_{B}\right]$ & -1 & -0.75 & 1 & -1.25 & 0.5 \\
\hline$s=3,\left[c_{E}, c_{B}, c_{A}, c_{D}, c_{C}\right]$ & -1.25 & -1.5 & 1 & -0.75 & 2 \\
\hline$s=4,\left[c_{E}, c_{C}, c_{A}, c_{B}, c_{D}\right]$ & -0.75 & 0 & 0.5 & -1 & 1.25 \\
\hline$s=5,\left[c_{A}, c_{B}, c_{E}, c_{D}, c_{C}\right]$ & -0.5 & -1 & 0.25 & -1.25 & 0.5 \\
\hline$s=6,\left[c_{D}, c_{A}, c_{B}, c_{C}, c_{E}\right]$ & -1.25 & -1 & 1.5 & -2 & 1.75 \\
\hline$s=7,\left[c_{C}, c_{E}, c_{B}, c_{D}, c_{A}\right]$ & -0.5 & 0.25 & 1 & -0.75 & 0.5 \\
\hline$s=8,\left[c_{D}, c_{B}, c_{E}, c_{A}, c_{C}\right]$ & -1.25 & -1 & 0.25 & -0.5 & -0.5 \\
\hline & $x_{1}$ & $x_{2}$ & $x_{3}$ & $x_{4}$ & $x_{5}$ \\
\hline allocation pattern & -1 & -1 & 1 & -1 & 1 \\
\cline { 2 - 6 }$\left[c_{1}, c_{2}, c_{3}, c_{4}, c_{5}\right]$ & $d_{1}$ & $d_{2}$ & $d_{3}$ & $d_{4}$ & $d 5$ \\
\hline$s=1,\left[c_{E}, c_{A}, c_{D}, c_{C}, c_{B}\right]$ & -0.25 & -0.5 & 0.75 & 0.5 & -1 \\
\hline$s=2,\left[c_{A}, c_{D}, c_{C}, c_{E}, c_{B}\right]$ & 1.5 & -1.25 & 1 & 0.75 & -1 \\
\hline$s=3,\left[c_{E}, c_{B}, c_{A}, c_{D}, c_{C}\right]$ & 1.25 & -0.5 & 1 & 0.75 & -1 \\
\hline$s=4,\left[c_{E}, c_{C}, c_{A}, c_{B}, c_{D}\right]$ & 2.25 & -1.5 & 1 & 1.5 & -1.25 \\
\hline$s=5,\left[c_{A}, c_{B}, c_{E}, c_{D}, c_{C}\right]$ & 1 & -0.5 & 1.25 & 0.75 & -1 \\
\hline$s=6,\left[c_{D}, c_{A}, c_{B}, c_{C}, c_{E}\right]$ & 1.25 & -1 & 0.5 & 1 & -1.75 \\
\hline$s=7,\left[c_{C}, c_{E}, c_{B}, c_{D}, c_{A}\right]$ & 1 & -1.75 & 0.5 & 1.25 & -1 \\
\hline$s=8,\left[c_{D}, c_{B}, c_{E}, c_{A}, c_{C}\right]$ & 0.75 & -0.5 & 1.25 & 1 & -1 \\
\hline
\end{tabular}

\begin{tabular}{|l|}
\hline$c_{A}=[-1,1,-1,1,-1,-1,-1,1,1,1,-1,1,1,1,-1,-1] / \sqrt{L}$ \\
\hline$c_{B}=[-1,1,-1,-1,-1,-1,1,-1,1,-1,1,-1,1,1,1,1] / \sqrt{L}$ \\
\hline$c_{C}=[-1,-1,-1,1,1,1,1,1,1,1,-1,1,-1,1,-1,1] / \sqrt{L}$ \\
\hline$c_{D}=[-1,-1,-1,1,1,-1,1,-1,-1,1,1,-1,1,-1,-1,-1] / \sqrt{L}$ \\
\hline$c_{E}=[1,1,1,-1,-1,-1,-1,1,1,-1,1,-1,1,1,-1,-1] / \sqrt{L}$ \\
\hline
\end{tabular}

and hence the users' cross-correlations, the decision variables are dramatically affected. It can also be viewed that some code allocations, e.g. $s=6, s=1$ for the first symbol combination, deliver better decision variables' distributions than others, e.g. $s=4, s=7$. As mentioned above this is a derivative of the difference in the resulting interference for different code allocations. Consequently the detection can be made more reliable by optimizing the code-to-user allocation to be employed at each symbol period. Additionally, it is apparent that for the second case, with a different symbol combination, different code allocations (e.g. $s=4, s=2$ ) provide improved decision variables' distributions. This is why in the proposed method the code allocation to be used is dynamically adjusted to the symbols $x_{i k}$ to be transmitted at the period $i$ of interest. This is a way of "fine tuning" the users' symbols and codes so that the energy in the channel be used constructively instead of being wasted because of data misalignment as in conventional methods. As a result the effective received SINR can be increased and improved decision variables can be delivered at the MUs' receivers without the need to increase the transmitted per-user-power.

\section{Code-to-User Allocation Method (CUA)}

In order to limit the amount of SI needed, the adaptive code-user allocation is performed by selecting the code-touser allocation for every symbol period from a limited number, $p_{c}$, of allocation patterns, each formed by shuffling $N_{c} \geq K$ spreading sequences amongst the users. The candidate patterns are known at both the transmitter and the receiver. By doing so, only the index of the allocation pattern needs to be conveyed 
to the receivers. The selected allocation pattern is chosen at the transmitter according to a certain optimization criterion which is explained in the subsection below.

\section{A. Method Analysis}

For reasons of simplicity, the analysis presented assumes Rake detection but the process for the case of Multiuser Detection (MUD) is easily deduced by analogy. In order to choose the appropriate code allocation pattern prior to transmission the expected decision variables at the MUs for all the available code-to-user allocation combinations need to be determined at the transmitter using the instantaneous symbol values for the active users. Towards this end, in the proposed method, the estimated effective crosscorrelation matrix $\widehat{R}_{s}$ of dimensions $K \times K$ is formed for each allocation pattern at the BS from the estimated $\widehat{\rho}_{k u}$ given by (6)-(7) in which the estimated channel coefficients $\widehat{h}_{p u}(i)$ are used

$$
\widehat{R}_{s}=\left[\begin{array}{cccc}
\widehat{\rho}_{11} & \widehat{\rho}_{12} & \cdots & \widehat{\rho}_{1 K} \\
\widehat{\rho}_{21} & \widehat{\rho}_{22} & \cdots & \widehat{\rho}_{2 K} \\
\vdots & \ddots & \ddots & \vdots \\
\widehat{\rho}_{K 1} & \widehat{\rho}_{K 2} & \cdots & \widehat{\rho}_{K K}
\end{array}\right] .
$$

The decision variables at the MUs outputs for the $i-t h$ symbol period for the $s-t h$ code-to-user allocation pattern can then be pre-estimated as

$$
\begin{aligned}
\widehat{d}_{i, s} & =\left[\begin{array}{llll}
\widehat{d}_{i 1, s} & \widehat{d}_{i 2, s} & \cdots & \widehat{d}_{i K, s}
\end{array}\right] \\
& =X_{i} A \widehat{R}_{s}=d_{i, s}+e_{i, s}
\end{aligned}
$$

where $X_{i}=\left[x_{i 1} x_{i 2} \ldots x_{i K}\right]$ is the $1 \times K$ data matrix for the $i$-th symbol period, $A=\operatorname{diag}\left(\left[a_{1} a_{2} \ldots a_{K}\right]\right)$ is the $K \times K$ diagonal matrix of amplitudes and $e_{i, s}$ is the decision variable pre-estimation error due to inaccurate channel estimation. It should be clarified that at each symbol period a new matrix $\widehat{d}_{i}$ is formed with the $s-t h$ row denoting the pre-estimated decision variables of the users for the $s-t h$ allocation pattern and the $u-t h$ column denoting the variables of the $u-t h$ user for the various allocation patterns. The proposed algorithm works as shown in the diagram in Fig. 1. Assuming $N_{c}=K$, at each symbol period and using the instantaneous values of $X_{i}$, the decision variables' distribution for each of the $p_{c}$ allocation patterns is evaluated using (9) and the optimal pattern is chosen according to a selection criteria that will be presented below. Since the receiver has knowledge of all the pc available patterns it only needs to be informed about the index of which specific pattern is used at each symbol-period by a control signal of $\left\lceil\log _{2}\left(p_{c}\right)\right\rceil$ bits transmitted at a different frequency/time slot as SI. By recognizing the correct pattern each MU can find the new code assigned to it for the current symbol detection as well as the remaining codes of the rest of the users to utilize for mutliuser detection (MUD).

An alternative route towards performance improvement, also considered here for the sake of comparison, is using a larger number of codes $\left(N_{c}>K\right)$ to attain a greater variety of interference distribution, but this would lead to the requirement for increased system resources, i.e. more available signature waveforms.

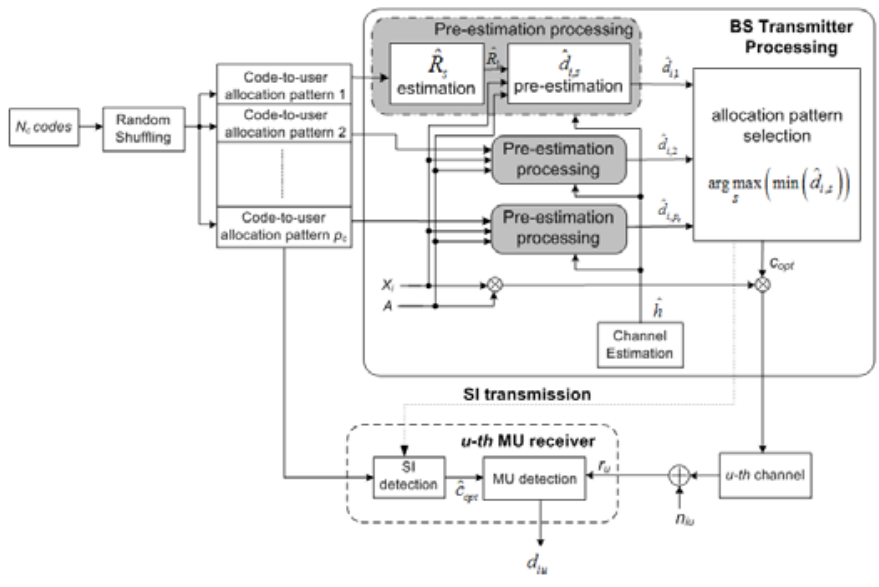

Fig. 1. Flow diagram of code allocation technique.

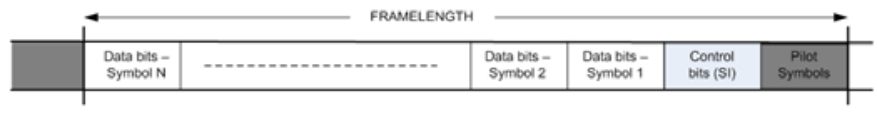

Fig. 2. Frame-based transmission structure for the proposed code allocation technique.

It is noteworthy to highlight that the SI bits are transmitted simultaneously and do not need to be spread as the information they convey is common to all users and furthermore this would be more bandwidth efficient. If the number of SI bits is not a power of two or if the SI is to be forward error correction encoded, then a frame based approach can be adopted as depicted in Fig. 2. Here the allocation procedure is run for all the symbols in the frame prior to transmission and the control bits for all symbols are transmitted in the beginning of the frame. Each symbol in the frame is a CDMA-multiplexed symbol for $K$ users.

\section{B. Allocation criteria}

It is intuitive from equation (9) and Table 1 that a number of criteria can be extracted for the selection of the optimum available code allocation pattern based on the instantaneous interference amongst users and the distribution of the resulting values of $\widehat{d}_{i}$. Since the performance of the worst user catalytically affects the overall system BER, the following code pattern selection criteria is proposed and examined here

$$
\underset{S}{\arg \max }\left(\min \left(\widehat{d}_{i, s}\right)\right) \text {. }
$$

In more detail, $\min \left(\widehat{d}_{i, s}\right)$ determines the MU output that is the most prone to decision errors for each code allocation. From the $p_{c}$ available distributions of $\widehat{d}_{i}$ according to the $p_{c}$ available code allocation patterns, the optimum is chosen as the one that maximizes the minimum of $\widehat{d}_{i}$ which denotes the decision variable of the worst user at each symbol period for each distribution of $\widehat{d}_{i}$. By maximizing the minimum of the decision variable distribution, the BER is minimized for the worst user. Hence, the code allocation selected $\left(c_{o p t}\right)$ is the one that delivers the highest decision variable for the worst user. In the case where two different allocation patterns offer the same minimum, the second minimum is considered and 


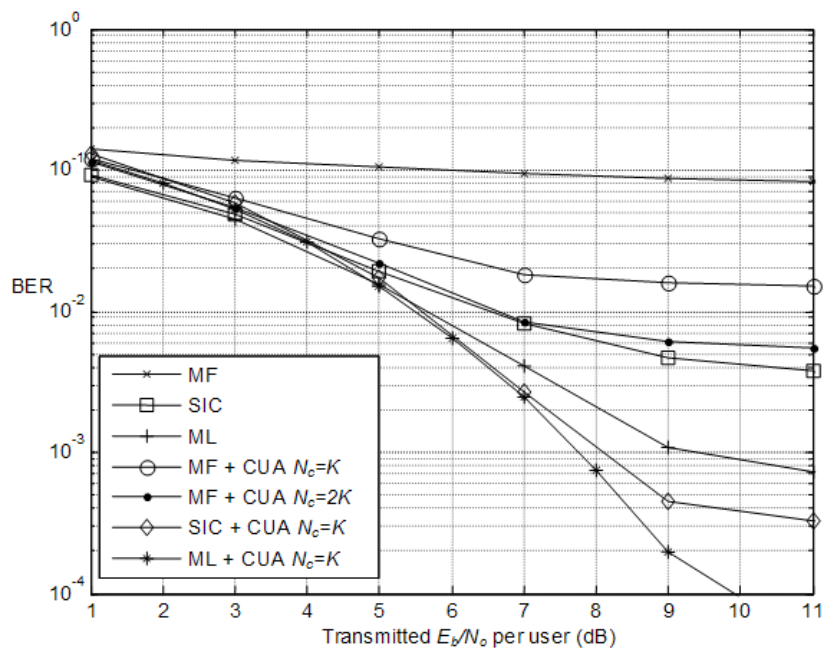

Fig. 3. Overall system BER for conventional and code allocation using MF, SIC, ML, with $N_{c}=K, 2 K, p_{c}=16, K=5, L=8$ in an AWGN channel.

so on. For the example depicted in Table 1 this criteria would indicate allocation pattern $s=3$ for the first symbol combination and pattern $s=4$ for the second. Evidently, this is one BER optimization approach that favors the users that are more susceptible to errors. By inspection of this criteria and Table 1 , the comparison to other available code allocation patterns shows that by this optimization a favorable constructive to destructive MAI ratio is chosen which evidently delivers a higher SINR and boosts performance. Constructive interference is enhanced while destructive interference is minimized.

\section{Numerical AND Simulation RESUlts}

Monte Carlo simulations for various combinations of the proposed technique with conventional methods have been performed for both flat and frequency selective fading channels with AWGN. Random codes of variable lengths have been used to allow for the worst data detection case and to illustrate performance improvement when conventional performance is poor. For the simulations in Figs. 3-5 the channels' characteristics are assumed to be perfectly known. For reasons of efficiency the SI bits are QPSK encoded. The BER shown in the figures is the average overall system BER in the systems considered. Performance of simple systems is investigated for reasons of reference, to illustrate the strength of the proposed scheme. Maximum likelihood (ML), successive interference cancellation (SIC) and matched filter (MF) detection with and without adaptive code-user allocation (CUA) are compared. However, the same comparison can be implemented with more advanced iterative Forward Error Correction (FEC)MUD receivers in more complex systems with higher numbers of users and longer codes. Since the proposed method aims at the enhancement and exploitation of constructive MAI it is intuitive and can be proven that significant performance benefits can still be offered. It should be noted that the Rake receiver analysis of (9) was extended to the other receiver types under investigation in order to attain the pre-estimated decision variables for the following simulations.

In Fig. 3 the performance of the proposed method with

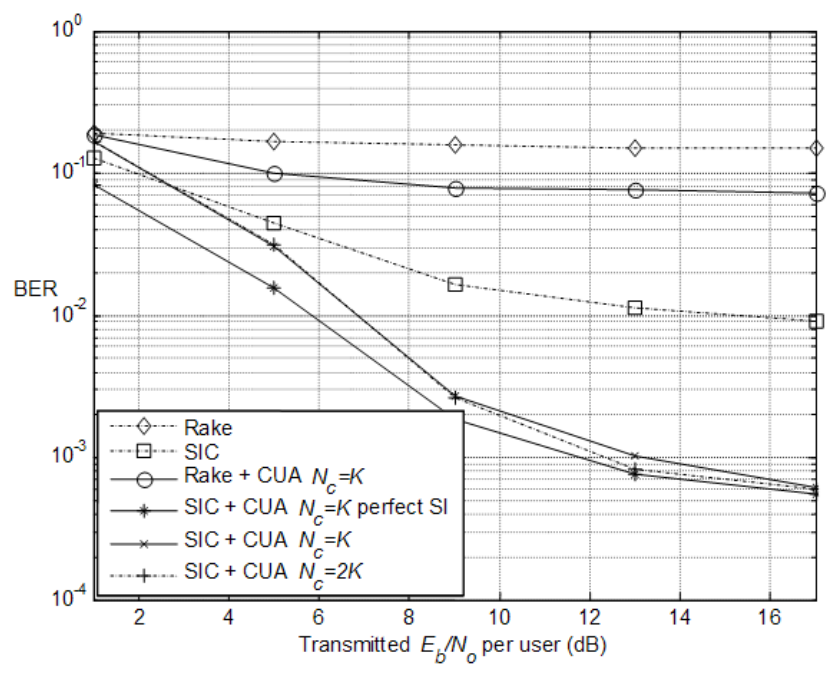

Fig. 4. Overall system BER performance of conventional Rake and SIC methods and the methods using the code allocation technique with $N_{c}=K$, $p_{c}=16$ in an Rayleigh multipath channel of $P=3$ with $K=20, L=31$.

$p_{c}=16$ allocation patterns formed for the cases of $N_{c}=K$ and $N_{c}=2 K$ codes in an AWGN channel is depicted. The number of users is $K=5$ and the spreading gain $L=8$. Performance of ML, successive SIC and MF detection with and without CUA are compared. It is evident in the figure that all three receiver structures benefit from a significant performance improvement when combined with the proposed technique. The fact that conventional ML is outperformed by SIC with adaptive code allocation even though it is the optimal MUD technique is not strange as it is due to the enhanced effective SINR that is attained with the adaptive allocation selection. It should be noted that using $N_{c}=2 \mathrm{~K}$ codes to form the possible allocation patterns adds no additional overhead to transmission compared to when $N_{c}=K$, since the number of the allocation patterns itself remains $p_{c}=16$. The difference is that there can be a greater variety of codes' cross-correlations and the decision variables can be optimized more efficiently. It is also evident that by using MF with adaptive code allocation and maintaining low complexity at the receiver the achieved performance is comparable to when employing conventional SIC with higher receiver complexity. It should be emphasized, however, that the effect of increasing $N_{c}$ becomes less significant as the number of users raises.

Fig. 4 depicts the comparison for the case of a Rayleigh frequency selective channel with $P=3$ paths for $K=20$ users and $p_{c}=16$ patterns formed from $N_{c}=K=20$ or $N_{c}=2 K=40$ codes of length $L=31$. Performance for Rake and SIC receiver schemes is depicted to show the proposed method's superiority. Simulation results with perfect SI detection for the proposed technique are also presented to show how the reliability of SI detection affects the overall performance.

Perfect SI detection refers to a genie-type detection of the SI bits. It can be seen that the results with imperfect SI detection converge to the case of perfect SI for high SNR as the SI detection becomes much more reliable. In all cases the proposed technique offers a significant performance enhancement. Obviously when the number of users is small the 


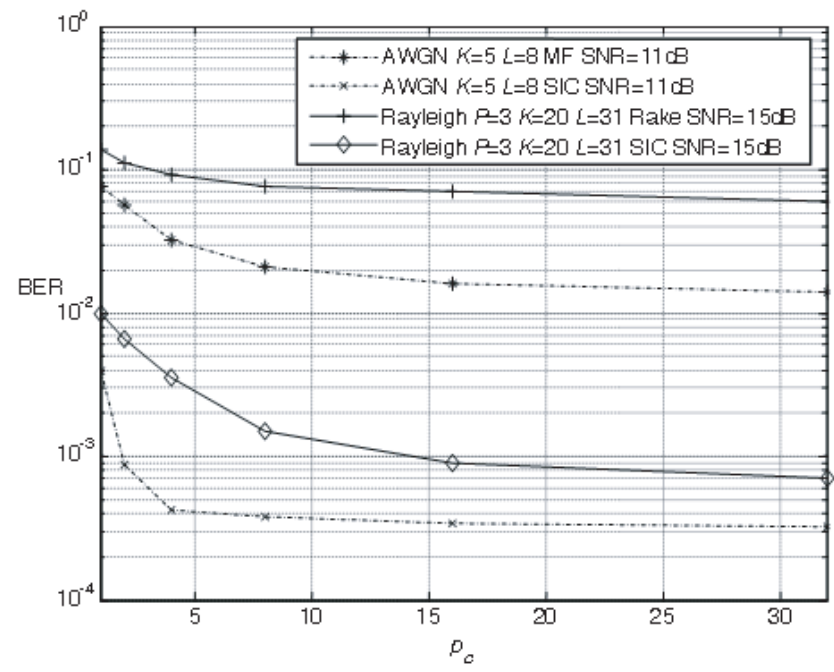

Fig. 5. Overall system BER vs. pc performance for the cases of $K=5$, $L=8$ in AWGN and $K=20, L=31$ in Rayleigh $P=3$ channel for MF and SIC detection employing code allocation for $\mathrm{SNR}=15 \mathrm{~dB}$.

number of side information bits (in this case $\left\lceil\log _{2}\left(p_{c}\right)\right\rceil=4$ equivalent to 2 QPSK symbols) may seem unacceptable, but for a more realistic number of active users such as 20 users the SI bits reduce the transmission rate efficiency to $20 / 22=91 \%$. Although a $9 \%$ efficiency reduction is not insignificant it is obvious from this figure that this method produces an overall system BER improvement of over an order of magnitude at $\mathrm{SNR}=17 \mathrm{~dB}$. Using a larger number of users or higher order modulation would further reduce the efficiency loss (e.g. in the case of 40 users or using 16PSK modulation the efficiency loss would be $\sim 5 \%$ ). It should be clarified that the above efficiency analysis is based on numbers of useful and SI symbols, as it is the most fair for both the cases when SI is transmitted in different time slots, as well as when transmitted on different frequencies. As regards increasing $N_{c}$, for the case of $N_{c}=2 \mathrm{~K}$ it can be seen that only a slight further performance improvement can be gained as already mentioned.

In Fig. 5 the overall system BER for different values of $p_{c}$ at $\mathrm{SNR}=11 \mathrm{~dB}$ is depicted for the system of Fig. 3 and at $\mathrm{SNR}=15 \mathrm{~dB}$ for the system of Fig. 4, to show the BER performance improvement as a function different values for $p_{c}$. For both systems increasing $p_{c}$ further than a specific value yields limited benefit. For $K=20, L=31$ the increase in $p_{c}$ up to the value of 32 brings smaller relative crosscorrelation variety improvement therefore the effect in performance is less significant. For $K=5, L=8$ the effect is more apparent because the increase in relative crosscorrelation variety is more drastic. This is because the number of all possible code allocation combinations is smaller and the number of $p_{c}=32$ patterns covers a higher percentage of it.

The case where imperfect CSI is available at the BS transmitter and MU receivers is considered in Fig. 6. Here, the deviation of the channel estimates from the actual channel coefficients is considered without limiting the performance to specific channel estimation schemes. Similarly to the evaluation method applied in [1], both real and imaginary parts of the estimated coefficients are deviated on average by

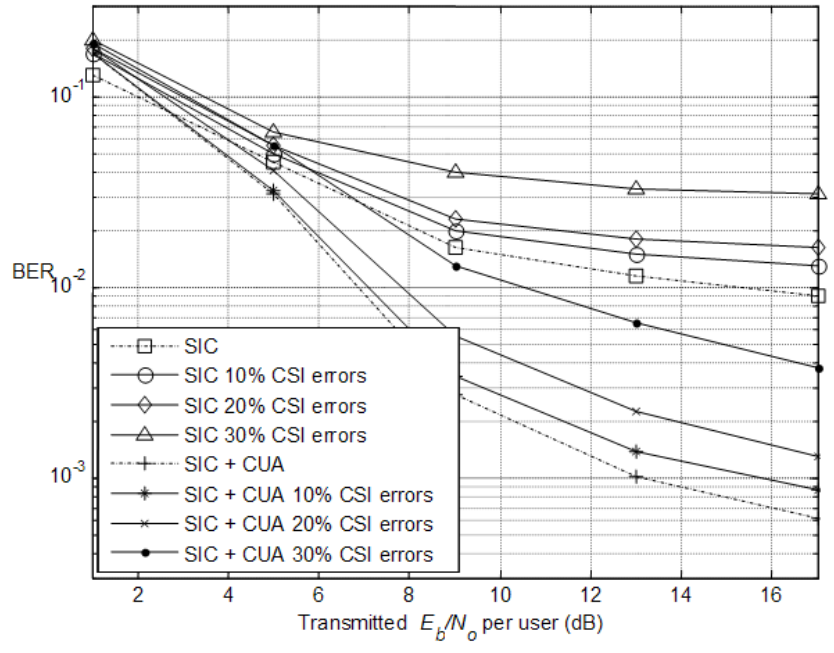

Fig. 6. Overall system BER performance of SIC methods and SIC-CUA with $N_{c}=K, p_{c}=16$ in an Rayleigh multipath channel of $P=3$ with $K=20, L=31$, with errors in the channel coefficients.

$\left|h_{p u}-\widehat{h}_{p u}\right| /\left|h_{p u}\right|=10,20$ and $30 \%$ of the actual channel values. SIC and SIC employing CUA are considered. The performance of the proposed scheme seems to deteriorate similarly to conventional performance for these considerable error percentages in CSI and at all cases SIC with CUA outperforms the conventional technique.

\section{CONCLUSION}

By "fine tuning" the users' codes so that the resulting decision variables at the MUs are optimized, we have shown that the proposed technique can significantly improve performance with no need for additional power-per-user investment. The trade-off is the need for transmission of control signals which imposes a small overhead on the system efficiency.

\section{ACKNOWLEDGMENT}

The authors would like to gratefully acknowledge the financial support for this work by EPSRC and Philips Research Labs UK, and express their thanks to Dr. Tim Moulsley for his invaluable advice.

\section{REFERENCES}

[1] M. Brandt-Pearce and A. Dharap, "Transmitter-based multiuser interference rejection for the down-link of a wireless CDMA system in a multipath environment," IEEE J. Select. Areas Commun., vol. 18, no. 3, pp. 407-417, Mar. 2000.

[2] G. W. Wornell, "Spread-signature CDMA: efficient multiuser communication in the presence of fading," IEEE Trans. Inform. Theory, vol. 41, pp. 1418-1438, Sept. 1995.

[3] W. H. Mow, "Minimizing the worst-case interuser interference experienced by any user in CDMA systems: a metric approach," in Proc. Int. Symp. Spread-Spectrum Techniques and Applications, vol. 2, Sept. 1996, pp. 561-565.

[4] B. R. Vojcic and W. M. Jang, "Transmitter precoding in synchronous multiuser communications," IEEE Trans. Commun., vol. 46, no. 10, pp. 1346-1355, Oct. 1998.

[5] G. J. R. Povey and M. Nakagawa, "A review of time division duplexCDMA techniques," ib Proc. Int. Symp. Spread-Spectrum Techniques and Applications, vol. 2, Sept. 1998, pp. 630-633. 